\title{
Diagnostic yield and safety of transbronchial cryobiopsy in sarcoidosis
}

\author{
Maria Jacob (101, Hélder Novais Bastos ${ }^{1,2,3}$, Patrícia Caetano Mota ${ }^{1,2}$, \\ Natália Melo ${ }^{1}$, Rui Cunha ${ }^{2,4}$, José Miguel Pereira ${ }^{2,4}$, Susana Guimarães ${ }^{2,5}$, \\ Conceição Souto Moura ${ }^{2,5}$ and António Morais (10 ${ }^{1,2,3}$
}

Affiliations: ${ }^{1}$ Pulmonology Dept, Centro Hospitalar Universitário de São João, Porto, Portugal. ${ }^{2}$ Faculty of Medicine of Porto University, Porto, Portugal. ${ }^{3}$ IBMC/i3S - Institute for Molecular and Cell Biology/Institute for Research and Innovation in Health, Porto, Portugal. ${ }^{4}$ Radiology Dept, Centro Hospitalar Universitário de São João, Porto, Portugal. ${ }^{5}$ Pathology Dept, Centro Hospitalar Universitário de São João, Porto, Portugal.

Correspondence: Maria Jacob, Pulmonology Dept, Centro Hospitalar Universitário de São João, Alameda Prof. Hernâni Monteiro, 4200-319 Porto, Portugal. E-mail: maria.gsgjđggmail.com

\section{ABSTRACT}

Introduction: Transbronchial lung cryobiopsy (TBLC) is an endoscopic technique proven to be useful in diagnostic approach to interstitial lung disease (ILD), but its role in sarcoidosis is not fully established. The aim of the present study was to assess the diagnostic yield of TBLC in sarcoidosis and its safety profile.

Methods: Retrospective analysis of patients, evaluated in a tertiary hospital ILD outpatient clinic, who underwent TBLC in the diagnostic work-up. TBLC was performed in accordance with the 2018 expert statement from the Cryobiopsy Working Group.

Results: 32 patients were included (mean \pm SD age $47.7 \pm 12.6$ years, $59.4 \%$ male) and divided into three groups: highly likely sarcoidosis $(n=21)$, possible sarcoidosis $(n=6)$ and unlikely sarcoidosis $(n=5)$. A mean of $2.8 \pm 0.8$ TBLCs were performed. The definitive diagnosis was established by TBLC in 20 out of 27 patients with suspected sarcoidosis. Two patients were diagnosed with sarcoidosis by other methods performed afterwards. TBLC leaded to other diagnosis as well, such as fungal infection $(n=1)$, hypersensitivity pneumonitis $(n=1)$ and silicosis $(n=3)$, making the diagnostic yield for suspected sarcoidosis of TBLC of $92.6 \%$. TBLC was also able to show compatible histological features in five patients whom sarcoidosis was not previously considered. The complications reported overall were pneumothorax in five $(15.6 \%)$ patients and moderate bleeding in one $(3.1 \%)$ case.

Conclusion: In this cohort, TBLC was a safe, reliable and useful procedure in sarcoidosis diagnosis. These results suggest that TBLC can be used successfully in those cases where a definitive diagnosis could not be reached with the usual and less-invasive diagnostic tools.

@ERSpublications

TBLC is a safe procedure with a high diagnostic yield in patients with a prior suspicion of sarcoidosis. Moreover, it can diagnose atypical cases in which previous methods are inconclusive. http://bit.ly/2KWJxLG

Cite this article as: Jacob M, Bastos HN, Mota PC, et al. Diagnostic yield and safety of transbronchial cryobiopsy in sarcoidosis. ERJ Open Res 2019; 5: 00203-2019 [https://doi.org/10.1183/ 23120541.00203-2019]. 


\section{Introduction}

Sarcoidosis is a multisystem inflammatory disorder of unknown aetiology characterised by the accumulation of macrophages and $\mathrm{CD} 4^{+}$T-lymphocytes in involved organs. It is associated with the formation of noncaseating granulomas and mainly affects the lungs, lymph nodes and skin [1-4].

The diagnosis of pulmonary sarcoidosis encloses a correct clinical setting, typical chest radiographic or high-resolution computed tomography (HRCT) features and a biopsy showing noncaseating granulomas [1-6]. Bronchoalveolar lavage fluid (BALF) is considered as a standard procedure in the diagnostic work-up of patients with interstitial lung diseases (ILDs). In sarcoidosis, the value of the BALF $\mathrm{CD} 4^{+} /$ $\mathrm{CD}^{+}$ratio has been examined by several authors, concluding that in a typical clinical scenario, a BALF $\mathrm{CD}^{+} / \mathrm{CD}^{+}$ratio $>3.5$ may support the diagnosis of sarcoidosis and obviate the need for a biopsy $[3,7,8]$.

However, a biopsy is quite often required, and preferentially, it should be performed non-invasively in peripheral structures, such as skin or conjunctiva [1-4]. Nevertheless, the lungs are affected in $>90 \%$ of the patients and frequently are the only organ involved and, therefore, the most frequently targeted for biopsies [1-4]. Traditionally, conventional transbronchial lung biopsy (TBLB), through bronchoscopy, was the elected method, with a variable diagnostic yield reported and with the disadvantages of small sample size and the occurrence of crush artefacts [4, 9-14]. More recently, endobronchial ultrasound (EBUS)-guided transbronchial needle aspirate (TBNA) in the patients with mediastinal adenopathies, namely Scadding stage I and II, which includes $\sim 70 \%$ of the patients, has significantly contributed to the pathologic support of sarcoidosis diagnosis $[15,16]$.

Since the first description of the potential use of transbronchial lung cryobiopsy (TBLC) in ILD by BABIAK et al. [17] in 2009, several authors have shown the feasibility of TBLC for obtaining adequate fragments of lung parenchyma [18-22]. Cryobiopsy fragments were larger than those obtained by TBLB, and do not contain relevant artefacts [17-23]. Furthermore, it is a safe procedure with a high overall diagnostic yield, typically $>80 \%$ in several datasets [17-23]. Although several series evaluating TBLC diagnostic yield contain patients with sarcoidosis, the data focusing on the potential role of this endoscopic technique in sarcoidosis are still scarce.

Concerning the regular use of TBLC in ILD multidisciplinary diagnosis, the aim of the present study was to find its relevance and safety, specifically in the diagnosis of sarcoidosis.

\section{Materials and methods}

Design

This retrospective study included patients who underwent TBLC in the bronchoscopy unit of the pulmonology department of Centro Hospitalar Universitário São João (Porto, Portugal), in the context of an ILD diagnostic work-up. Cases were monitored over 4 years (May 2014 to December 2017). The ethics committee approved this study.

\section{Patients}

All the patients had a prior evaluation at the ILD outpatient clinic. A detailed history, which included drug and occupational exposure, was taken and all the patients underwent a complete physical examination in addition to complete blood count, serum biochemistry and coagulation tests, lung function and arterial blood gas tests, thoracic HRCT, ECG and echocardiogram. The patients' electronic records were accessed retrospectively, and the following data were collected: age, sex, lung function tests and BALF results, radiographic data, procedure and pathology reports. Post-procedure complications and 30-day post-interventional morbidity and mortality were recorded.

Sarcoidosis diagnosis was based on a multimodality approach that combined clinical, radiological and histological evaluation showing noncaseating granulomas, according to American Thoracic Society (ATS)/ European Respiratory Society (ERS)/World Association of Sarcoidosis and Other Granulomatous Disorders (WASOG) criteria [3]. When the diagnosis of sarcoidosis was considered, all patients underwent bronchoscopy with BALF (according to the ERS recommendations [24]) and EBUS-TBNA, specifically in patients classified by the Scadding criteria as stage I and II. Patients were discussed and proposed for TBLC, in the multidisciplinary diagnosis team meeting, only when clinical and radiological findings, BALF features and EBUS-TBNA cytological specimens did not conclude a final diagnosis, according ERS/ATS/ WASOG criteria [3]. Exclusion criteria included the usual contraindications for TBLC [5]: a platelet count of $<70000$, an international normalised ratio of $>1.5$ or activated partial thromboplastin time of $>50 \mathrm{~s}$, forced vital capacity (FVC) of $<50 \%$ predicted, a forced expiratory volume in $1 \mathrm{~s}\left(\mathrm{FEV}_{1}\right)$ of $<80 \%$ pred, a diffusing capacity of the lung for carbon monoxide $\left(D_{\mathrm{LCO}}\right)$ of $<35 \%$ pred, a partial pressure of oxygen in arterial blood of $<55 \mathrm{mmHg}$, diffuse bullous disease, uncontrolled heart disease or a transthoracic echocardiogram-estimated pulmonary artery systolic pressure of $>40 \mathrm{mmHg}$. 
Patients were stratified into three groups, according to diagnostic suspicion previous to TBLC: highly likely sarcoidosis (HLS), due to typical clinical and radiological features; possible sarcoidosis, based on a differential diagnosis that included sarcoidosis; and unlikely sarcoidosis (ULS), for patients whose clinical presentations and/or radiological appearance were not suggestive, but at the end TBLC showed histological features of sarcoidosis.

\section{Procedure}

TBLC was performed according to 2018 expert statement from the Cryobiopsy Working Group [25]. TBLC was performed using a combination of rigid bronchoscopy (Tracheoscope $14 \mathrm{~mm}$; Karl Storz, Tuttingen, Germany) and flexible bronchoscopy (Olympus BF-XT40; Hamburg, Germany), under general anaesthesia with manual jet ventilation (working pressure of $\sim 2 \mathrm{bar}$ ). All the steps to the procedure were performed as previously described by ALMEIDA et al. [19].

Following the procedure, patients were extubated and kept under observation. After $3 \mathrm{~h}$, chest radiography was performed to exclude pneumothorax, and the patient was discharged. Complications such as iatrogenic pneumothorax, endobronchial bleeding and 30-day post-interventional morbidity and mortality were documented. Pneumothorax was described according to observation measures or chest tube insertion requirements. Endobronchial bleeding was classified using the British Thoracic Society system: mild bleeding (continued suctioning of blood from the airways, bleeding stops spontaneously); moderate bleeding (intubation of the biopsied segment with the bronchoscope into the wedge position, use of adrenaline or cold saline to stop bleeding); and severe bleeding (placement of bronchus blocker or catheter, applying fibrin sealant, resuscitation, blood transfusion, admission to critical care unit or death).

Written informed consent was obtained, before TBLC, from all patients. Given that the investigation was limited to the review of existing medical records, the requirement for an additional written consent was waived for the study purposes. To ensure confidentiality, each case was anonymised by the assignment of a random identification number.

\section{Pathologic assessment}

The sample tissue was formalin-fixed for $\geqslant 6 \mathrm{~h}$ and at most $24 \mathrm{~h}$ before paraffin embedding. Sections measuring $3 \mu \mathrm{m}$ were stained with haematoxylin-eosin. In all cases, serial cuts were made at three levels, with the additional use of special stains or immunohistochemical stains when necessary. All samples were evaluated under the microscope, and the area of each fragment measured. The presence of pleural tissue was recorded. The biopsy was considered adequate if at least one of the fragments was constituted by alveolated lung parenchyma. The diagnosis was made based on the same criteria used for surgical lung biopsy (SLB).

\section{Statistical analysis}

Descriptive statistical measures of the studied variables included mean $\pm \mathrm{sD}$ for continuous variables and $\mathrm{n}$ (\%) for each category of categorical variables. The diagnostic sensitivity, specificity and accuracy for TBLC were calculated. Statistical analysis was performed using the SPSS software programme (version 25; IBM, Armonk, NY USA).

\section{Results}

Between May 2014 and December 2017, 32 patients were eligible for the current study. Their mean age was $47.7 \pm 12.6$ years, and $19(59.4 \%)$ of the patients were male. The mean FVC was $98.3 \pm 21.5 \%$ pred, $\mathrm{FEV}_{1}$ was $94.4 \pm 21.2 \%$ pred and $D_{\mathrm{LCO}}$ was $76.3 \pm 18.6 \%$ pred. BALF was performed on all patients, with a mean lymphocyte count of $33.7 \pm 16.2 \%$ and a mean CD4/CD8 ratio of $3.2 \pm 3.0$ (table 1 ).

A mean 2.8 \pm 0.8 TBLCs in different segments were performed for each patient. The upper right lobe was assessed in 11 (34.4\%) cases, the inferior right lobe in $10(31.3 \%)$ cases, the upper left lobe in seven $(21.9 \%)$ cases and inferior left lobe in one $(3.1 \%)$ case. In three $(9.3 \%)$ cases, the biopsies were performed in different segments of two lobes. All the samples were considered adequate. The mean sample length was $5.5 \pm 1.2 \mathrm{~mm}$, and the mean area was $16.3 \pm 4.2 \mathrm{~mm}^{2}$.

21 patients from the HLS group, six patients from the possible sarcoidosis group and five from the ULS group underwent TBLC. Figure 1 summarises the overall yield of the study population. Of the HLS group $(\mathrm{n}=21)$, TBLC was able to confirm the diagnosis in $17(81 \%)$ patients. According to the Scadding radiological criteria, eight patients were stage II (six had previous negative EBUS-TBNA), eight patients were stage III and one patient was stage IV. Sarcoidosis was subsequently confirmed by transthoracic lung biopsy in two patients with stage III who had presented unspecific features at TBLC. In a patient with recurrent uveitis and random micronodules in the lung parenchyma, TBLC showed histological features of 


\begin{tabular}{|c|c|c|c|c|}
\hline & Total & HLS & PS & ULS \\
\hline Male & $19(59.4)$ & $11(52.4)$ & $6(100)$ & $2(40)$ \\
\hline Age years & $47.7 \pm 12.6$ & $44.5 \pm 11$ & $46 \pm 11.3$ & $62.8 \pm 11.1$ \\
\hline \multicolumn{5}{|l|}{ Pulmonary function test } \\
\hline FVC \% pred & $98.3 \pm 21.5$ & $92.1 \pm 18.6$ & $105.6 \pm 22.2$ & $115.5 \pm 24.5$ \\
\hline $\mathrm{FEV}_{1} \%$ pred & $94.4 \pm 21.2$ & $87.7 \pm 17.5$ & $105.3 \pm 24.1$ & $109.7 \pm 22.8$ \\
\hline $\mathrm{FEV}_{1} / \mathrm{FVC}$ & $77.9 \pm 7.0$ & $77.4 \pm 8.2$ & $79.8 \pm 4.9$ & $77.5 \pm 3.8$ \\
\hline$D_{\text {Lco }} \%$ pred & $76.3 \pm 18.6$ & $72.5 \pm 17.1$ & $91 \pm 19$ & $79.3 \pm 21.2$ \\
\hline$D_{\mathrm{LCO}} / V_{\mathrm{A}} \%$ pred & $88.6 \pm 18.8$ & $84.3 \pm 13.5$ & $112.5 \pm 22.6$ & $85 \pm 21.3$ \\
\hline \multicolumn{5}{|l|}{ BAL results } \\
\hline Lymphocytes \% & $33.7 \pm 16.2$ & $37.2 \pm 16.3$ & $22.9 \pm 14.4$ & $32 \pm 14$ \\
\hline Neutrophils \% & $2.9 \pm 3.2$ & $2.1 \pm 1.7$ & $5.6 \pm 5.9$ & $3 \pm 2.5$ \\
\hline Eosinophil \% & $0.5 \pm 0.7$ & $0.4 \pm 0.7$ & $0.7 \pm 0.7$ & $0.6 \pm 0.7$ \\
\hline CD4/CD8 ratio & $3.2 \pm 3.0$ & $3.3 \pm 3.5$ & $3.4 \pm 1.7$ & $2 \pm 1.3$ \\
\hline \multicolumn{5}{|l|}{ Chest $\mathrm{CT}$ findings } \\
\hline Micronodularity & $25(78.1)$ & $17(81)$ & $6(100)$ & $2(40)$ \\
\hline Adenopathy & 10 (31.3) & 9 (42.9) & $1(16.7)$ & 0 \\
\hline Consolidation & $2(6.3)$ & $2(9.5)$ & 0 & 0 \\
\hline Air trapping/mosaic pattern & $2(6.3)$ & 0 & 0 & $2(40)$ \\
\hline Ground glass & $2(6.3)$ & $1(4.8)$ & 0 & $1(20)$ \\
\hline Fibrosis & $1(3.2)$ & $1(4.8)$ & 0 & 0 \\
\hline \multicolumn{5}{|c|}{$\begin{array}{l}\text { Data are presented as } \mathrm{n}(\%) \text { or mean } \pm \mathrm{SD} \text {. HLS: highly likely sarcoidosis; PS: possible sarcoidosis; ULS: } \\
\text { unlikely sarcoidosis; FVC: forced vital capacity; } \mathrm{FEV}_{1} \text { : forced expiratory volume in } 1 \mathrm{~s} ; D_{\mathrm{LCO}} \text { : diffusing } \\
\text { capacity of the lung for carbon monoxide; } D_{\mathrm{LCO}} / V_{\mathrm{A}}: D_{\mathrm{LCO}} \text { adjusted for alveolar volume; BAL: } \\
\text { bronchoalveolar lavage; CT: computed tomography. }\end{array}$} \\
\hline
\end{tabular}

hypersensitivity pneumonitis. The remaining patient had mediastinal adenopathies and bilateral consolidations, and TBLC showed the characteristic features of a fungal infection.

From the group of patients with possible sarcoidosis $(n=6)$, TBLC was able to establish this diagnosis in three $(50 \%)$ patients. One patient had mediastinal adenopathies, and all of them had parenchymal random micronodules (one patient also had bilateral consolidations). In the remaining three cases, TBLC showed histological features that were compatible with silicosis, leading to a definitive diagnosis. In five patients whose sarcoidosis was not considered previously due to atypical findings, TBLC showed histological features that led to the definitive diagnosis of sarcoidosis. None of these patients had radiological adenopathies, and in the lung parenchyma, two patients presented a predominantly mosaic pattern, two patients had scarce peripheric nodules and one presented a diffuse ground-glass pattern.

TBLC established the definitive diagnosis of sarcoidosis in 20 out of the 27 patients with suspected sarcoidosis, and only two patients were diagnosed with sarcoidosis by other methods that were performed afterwards. In addition, TBLC was able to diagnose a fungal infection $(\mathrm{n}=1)$, hypersensitivity pneumonitis $(n=1)$ and silicosis $(n=3)$. As such, the diagnostic yield of TBLC was $92.6 \%$, its sensitivity was $90.9 \%$ and its specificity was $100 \%$. In this series, TBLC was able to diagnose sarcoidosis in five patients for whom the diagnosis had not been considered previously.

Pneumothorax was reported in five (15.6\%) patients, and four of these required drainage (mean 3 days, maximum 4 days). While there were several cases of mild bleeding, these were considered to be a normal event with this procedure. Moderate bleeding was reported in one $(3.13 \%)$ case. No severe bleeding was noticed. Lastly, no deaths occurred during TBLC or the 30-day post-intervention.

\section{Discussion}

In the current study, TBLC resulted in a high diagnostic yield in patients with clinical and radiological suspicion of sarcoidosis not previously diagnosed with less invasive procedures such as BALF and/or EBUS-TBNA. The definitive diagnosis was established by TBLC in $92.6 \%$ of these patients, enabling a complete histological diagnosis not only in patients who displayed typical CT patterns, but also in others with more heterogeneous CT patterns such as ground glass, air trapping/mosaic or consolidation.

A diagnosis of sarcoidosis is most likely to be accurate if clinical and radiological data are supported by the demonstration of noncaseating granulomas or by the exclusion of other diseases known to cause 


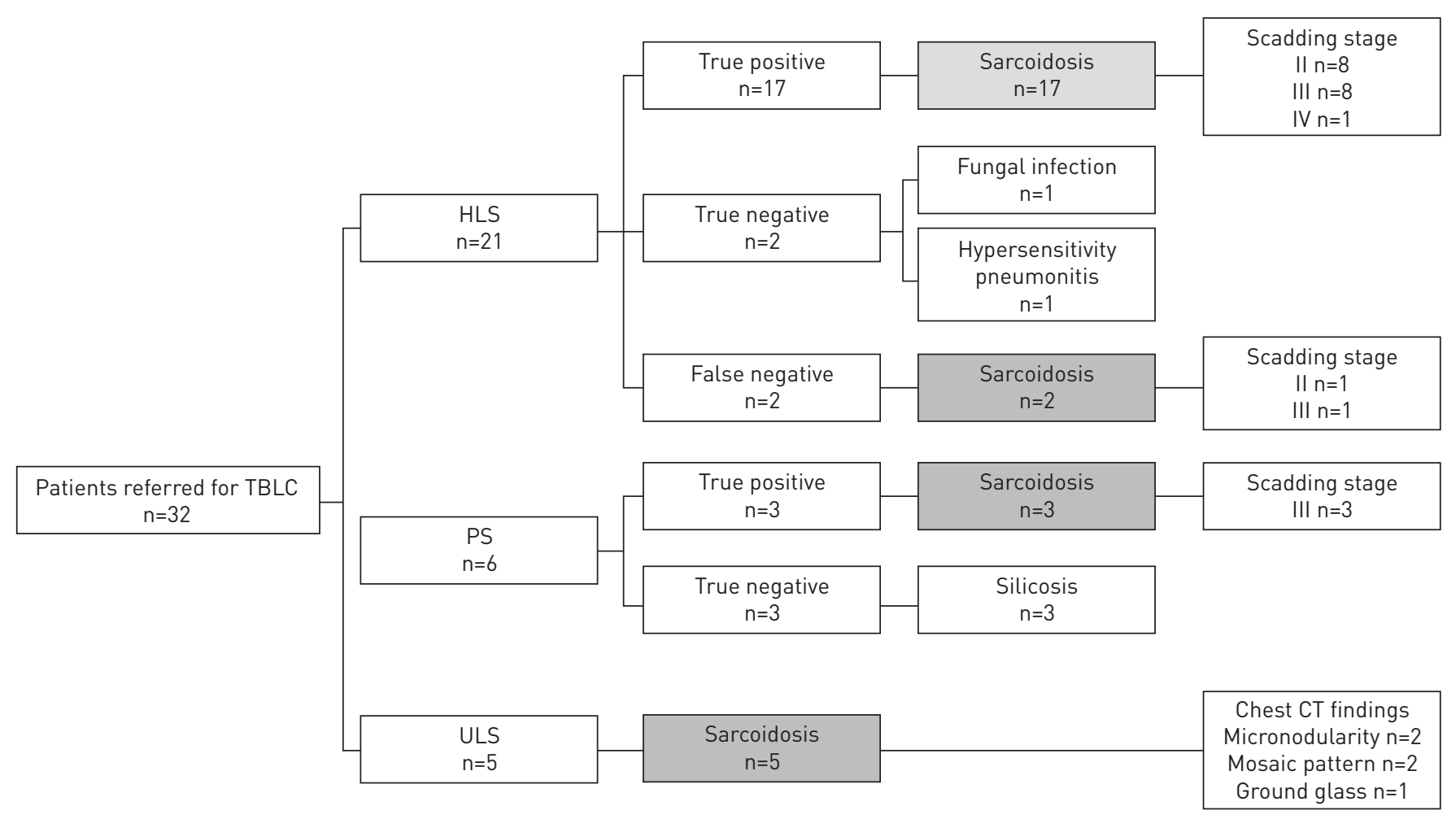

FIGURE 1 Diagrammatic representation of the diagnostic performance of transbronchial lung cryobiopsy. HLS: highly likely sarcoidosis; PS: possible sarcoidosis; ULS: unlikely sarcoidosis; CT: computed tomography.

granulomatous disease $[1-4,12]$. The best diagnostic approach varies substantially depending on the clinical scenario [4]. Because pulmonary involvement is the most common presentation in sarcoidosis, diagnostic procedures at bronchoscopy can help to achieve a histological confirmation $[3,4,12]$. While in a typical clinicoradiological scenario, a BALF $\mathrm{CD}^{+} / \mathrm{CD}^{+}$ratio of $>3.5$ is considered sufficient for establishing a definitive diagnosis, only $\sim 50-60 \%$ of cases present all of these features $[8,15]$. In 1999 , the ATS/ERS/WASOG statement on sarcoidosis recommended TBLB as the first choice for tissue sampling [3]. Although a safe and straightforward procedure, performed during a conventional bronchoscopy, where BALF and bronchial biopsies can also be performed, the samples obtained are small and usually present crush artefacts that negatively impact the integrity and quality of the sample. Regarding its diagnostic yield in sarcoidosis, studies vary from as much as $40 \%$ to $>90 \%$, depending on the number of biopsies performed and the level of bronchoscopy expertise [9-14, 26, 27].

With the availability of real-time evaluation by ultrasound, EBUS-TBNA has led to a decreased use of conventional transbronchial needle aspiration in the past decade. Several studies and meta-analyses have shown that EBUS-TBNA is a safe bronchoscopic technique with high overall accuracy for diagnosis [28-32]. In a randomised clinical trial [15], among patients with suspected stage I or II pulmonary sarcoidosis, the use of endosonography resulted in a diagnostic yield of $80 \%$, which was higher than the diagnostic yield of 53\% for TBLB and bronchial biopsy. Tournoy et al. [33] showed, in a prospective study, that endosonography had a high yield in diagnosing sarcoidosis after a previous nondiagnostic bronchoscopy. Accordingly, nowadays, EBUS-TBNA is a standard procedure in the diagnostic process of sarcoidosis with thoracic involvement and mediastinal adenopathies.

Currently, TBLC is gaining momentum in the diagnosis of sarcoidosis. In several series, TBLC has proven to be useful in diagnosing various ILDs, including sarcoidosis [18, 20, 22, 34-37], with lower complication and mortality rates than SLB. TBLC can retrieve larger biopsies and preserve architecture (avoiding the crushing effect of a TBLB). In a recent review of bronchoscopic modalities for the diagnosis of sarcoidosis [12], the authors proposed a diagnostic flowchart where they recommended the upfront execution of BALF for all patients with or without hilar/mediastinal adenopathy, followed by endobronchial biopsies, with or without endobronchial sarcoidosis features. To improve the diagnostic yield, they also recommended either TBLC or TBLB in patients with parenchymal disease.

In the present study, the diagnostic yield for suspicion of sarcoidosis was $92.6 \%$ with TBLC, with a sensitivity of $90.9 \%$. This diagnostic yield was higher than the majority of the data described for TBLB. 
ARAGAKI-NAKAHODO et al. [36] compared the diagnostic yield of TBLC and EBUS-TBNA in 36 patients with suspected sarcoidosis. For patients with a pathological diagnosis of sarcoidosis, the overall yield for TBLC and EBUS-TBNA was $67 \%$, for each procedure individually, increasing to $100 \%$ when the procedures were combined. The authors agreed that TBLC increased the yield and was specifically helpful in cases with parenchymal abnormalities and with/without significant adenopathy. In this regard, the value of combining different bronchoscopic techniques to increase the diagnostic yield is not novel and has been portrayed in previous works, including in a recent meta-analysis [13]. In another study [38], for patients who had either declined or had a non-functional status for SLB, TBLC achieved a high diagnostic yield, with most frequent histopathological finding being sarcoidosis, proving that this technique could be an alternative method for diagnosis in patients unfit for SLB. Several series on ILD patients who were submitted to TBLC have described patients with a final diagnosis of sarcoidosis; however, specific data about the diagnostic yield and the complications of this particular setting were not discussed [18-22]. The present study adopted a different approach, establishing its criteria on the levels of suspicion. The overall strategy, based on the initial less-invasive bronchoscopy techniques, such as BALF in some, with additional EBUS-TBNA, followed by TBLC in those cases without a final diagnosis with the previous procedures, led to a high diagnostic yield. In atypical cases, TBLC provides larger and adequate tissue samples associated with high diagnostic yield, thereby avoiding SLB significantly.

In cases with characteristic clinical and radiological sarcoidosis findings, we usually only freeze for $3 \mathrm{~s}$ when TBLC is taken, as usually there is no need for larger biopsies, as in fibrotic interstitial pneumonia. This conservative approach resulted in a lower mean area of $16.3 \mathrm{~mm}^{2}$ than the overall mean area of $22.65 \mathrm{~mm}^{2}$ for the TBLCs evaluated in the same period at our research centre.

Similar to the series by ARAGAKI-NAKAHODO et al. [36], pneumothorax was reported in $15.6 \%$ of patients and moderate bleeding in $3.1 \%$. Although easily managed with a chest tube and a short inpatient stay, pneumothorax was a significant adverse event. Since this sample represents the initial 200 patients submitted to TBLC in our centre, the authors believe that a larger cohort and adding to the learning curve of TBLC, the number of pneumothoraxes would be lower. We have previously described that TBLC proficiency was achieved after $\sim 70$ procedures, with lower complication rate, underscoring the importance of experience by TBLC technicians [19].

Due to its retrospective nature, one of the main limitations is the inability to compare different endoscopic techniques to TBLC. Moreover, this report represents the experience of one centre, so data inevitably reflects its specific clinical and technical methodology. As an example, bronchial biopsy was only performed in cases with endobronchial alterations, which challenges several opinions in literature defending that bronchial biopsy should be performed in all cases of sarcoidosis suspicion. This cohort did not include patients with endobronchial abnormalities, thus bronchial biopsies were not performed. It can also be argued that a previous TBLB could have provided the final diagnosis in some patients included in this series. However, due to the larger sample dimension, accuracy and diagnostic yield of TBLC in comparison with TBLB, the latter has been seldom performed in our centre.

In conclusion, the high diagnostic yield was found for TBLC in patients with a prior suspicion of sarcoidosis. In this instance, TBLC could be complementary to other sampling modalities and could prove to be especially helpful in cases without significant adenopathies and parenchymal abnormalities. Moreover, in atypical cases, when previous diagnostic methods are inconclusive, TBLC could avoid SLB, which are associated with higher rates of morbidity and mortality, higher costs and longer hospital stays than TBLC. In the future, multicentre and prospective studies will be needed to better understand TBLC role and accuracy in the diagnostic specificity of sarcoidosis.

Conflict of interest: None declared.

\section{References}

Valeyre D, Prasse A, Nunes H, et al. Sarcoidosis. Lancet 2014; 383: 1155-1167.

Baughman RP, Culver DA, Judson MA. A concise review of pulmonary sarcoidosis. Am J Respir Crit Care Med 2011; 183: 573-581.

3 Hunninghake GW, Costabel U, Ando M, et al. ATS/ERS/WASOG statement on sarcoidosis. American Thoracic Society/European Respiratory Society/World Association of Sarcoidosis and other Granulomatous Disorders. Sarcoidosis Vasc Diffuse Lung Dis 1999; 16: 149-173.

4 Spagnolo P, Rossi G, Trisolini R, et al. Pulmonary sarcoidosis. Lancet Respir Med 2018; 6: 389-402.

5 Valeyre D, Bernaudin JF, Uzunhan Y, et al. Clinical presentation of sarcoidosis and diagnostic work-up. Semin Respir Crit Care Med 2014; 35: 336-351.

6 Judson MA. The diagnosis of sarcoidosis. Clin Chest Med 2008; 29: 415-427.

7 Meyer KC, Raghu G, Baughman RP, et al. An official American Thoracic Society clinical practice guideline: the clinical utility of bronchoalveolar lavage cellular analysis in interstitial lung disease. Am J Respir Crit Care Med 2012; 185: 1004-1014. 

sarcoidosis. Respir Med 2012; 106: 1014-1020.

9 Hsu RM, Connors AF Jr, Tomashefski JF Jr. Histologic, microbiologic, and clinical correlates of the diagnosis of sarcoidosis by transbronchial biopsy. Arch Pathol Lab Med 1996; 120: 364-368.

10 Oki M, Saka H, Kitagawa C, et al. Prospective study of endobronchial ultrasound-guided transbronchial needle aspiration of lymph nodes versus transbronchial lung biopsy of lung tissue for diagnosis of sarcoidosis. $J$ Thorac Cardiovasc Surg 2012; 143: 1324-1329.

11 Mall W. Transbronchoscopic lung biopsy in sarcoidosis. Bronchopneumologie 1980; 30: 331-335.

12 Benzaquen S, Aragaki-Nakahodo AA. Bronchoscopic modalities to diagnose sarcoidosis. Curr Opin Pulm Med 2017; 23: 433-438.

13 Goyal A, Gupta D, Agarwal R, et al. Value of different bronchoscopic sampling techniques in diagnosis of sarcoidosis: a prospective study of 151 patients. J Bronchology Interv Pulmonol 2014; 21: 220-226.

14 Halme M, Piilonen A, Taskinen E. Comparison of endobronchial and transbronchial biopsies with high-resolution CT (HRCIT) in the diagnosis of sarcoidosis. APMIS 2001; 109: 289-294.

15 von Bartheld MB, Dekkers OM, Szlubowski A, et al. Endosonography vs conventional bronchoscopy for the diagnosis of sarcoidosis: the GRANULOMA randomized clinical trial. JAMA 2013; 309: 2457-2464.

16 Neves I, Sucena M, Magalhães A, et al. EBUS in pulmonary sarcoidosis: what to expect? Rev Port Pneumol 2014; 20: 229-231.

17 Babiak A, Hetzel J, Krishna G, et al. Transbronchial cryobiopsy: a new tool for lung biopsies. Respiration 2009; 78 203-208

18 Casoni GL, Tomassetti S, Cavazza A, et al. Transbronchial lung cryobiopsy in the diagnosis of fibrotic interstitial lung diseases. PLoS One 2014; 9: e86716.

19 Almeida LM, Lima B, Mota PC, et al. Learning curve for transbronchial lung cryobiopsy in diffuse lung disease. Rev Port Pneumol 2018; 24: 23-31.

20 Dhooria S, Sehgal IS, Aggarwal AN, et al. Diagnostic yield and safety of cryoprobe transbronchial lung biopsy in diffuse parenchymal lung diseases: systematic review and meta-analysis. Respir Care 2016; 61: 700-712.

21 Johannson KA, Marcoux VS, Ronksley PE, et al. Diagnostic yield and complications of transbronchial lung cryobiopsy for interstitial lung disease. A systematic review and metaanalysis. Ann Am Thorac Soc 2016; 13: $1828-1838$.

22 Ravaglia C, Bonifazi M, Wells AU, et al. Safety and diagnostic yield of transbronchial lung cryobiopsy in diffuse parenchymal lung diseases: a comparative study versus video-assisted thoracoscopic lung biopsy and a systematic review of the literature. Respiration 2016; 91: 215-227.

23 Colella S, Haentschel M, Shah $\mathrm{P}$, et al. Transbronchial lung cryobiopsy in interstitial lung diseases: best practice. Respiration 2018; 95: 383-391.

24 Technical recommendations and guidelines for bronchoalveolar lavage (BAL). Report of the European Society of Pneumology Task Group. Eur Respir J 1989; 2: 561-585.

25 Hetzel J, Maldonado F, Ravaglia C, et al. Transbronchial cryobiopsies for the diagnosis of diffuse parenchymal lung diseases: expert statement from the cryobiopsy working group on safety and utility and a call for standardization of the procedure. Respiration 2018; 95: 188-200.

26 Agarwal R, Aggarwal AN, Gupta D. Efficacy and safety of conventional transbronchial needle aspiration in sarcoidosis: a systematic review and meta-analysis. Respir Care 2013; 58: 683-693.

27 Gupta D, Dadhwal DS, Agarwal R, et al. Endobronchial ultrasound-guided transbronchial needle aspiration vs conventional transbronchial needle aspiration in the diagnosis of sarcoidosis. Chest 2014; 146: 547-556.

28 Wong M, Yasufuku K, Nakajima T, et al. Endobronchial ultrasound: new insight for the diagnosis of sarcoidosis Eur Respir J 2007; 29: 1182-1186.

29 Garwood S, Judson MA, Silvestri G, et al. Endobronchial ultrasound for the diagnosis of pulmonary sarcoidosis. Chest 2007; 132: 1298-1304.

30 Nakajima T, Yasufuku K, Kurosu K, et al. The role of EBUS-TBNA for the diagnosis of sarcoidosis - comparisons with other bronchoscopic diagnostic modalities. Respir Med 2009; 103: 1796-1800.

31 Agarwal R, Srinivasan A, Aggarwal AN, et al. Efficacy and safety of convex probe EBUS-TBNA in sarcoidosis: a systematic review and meta-analysis. Respir Med 2012; 106: 883-892.

32 Trisolini R, Lazzari Agli L, Tinelli C, et al. Endobronchial ultrasound-guided transbronchial needle aspiration for diagnosis of sarcoidosis in clinically unselected study populations. Respirology 2015; 20: 226-234.

33 Tournoy KG, Bolly A, Aerts JG, et al. The value of endoscopic ultrasound after bronchoscopy to diagnose thoracic sarcoidosis. Eur Respir J 2010; 35: 1329-1335.

34 Pajares V, Puzo C, Castillo D, et al. Diagnostic yield of transbronchial cryobiopsy in interstitial lung disease: a randomized trial. Respirology 2014; 19: 900-906.

35 Kropski JA, Pritchett JM, Mason WR, et al. Bronchoscopic cryobiopsy for the diagnosis of diffuse parenchyma lung disease. PLoS One 2013; 8: e78674.

36 Aragaki-Nakahodo AA, Baughman RP, Shipley RT, et al. The complimentary role of transbronchial lung cryobiopsy and endobronchial ultrasound fine needle aspiration in the diagnosis of sarcoidosis. Respir Med 2017; 131: 65-69.

37 Griff S, Schönfeld N, Ammenwerth W, et al. Diagnostic yield of transbronchial cryobiopsy in non-neoplastic lung disease: a retrospective case series. BMC Pulm Med 2014; 14: 171.

38 Sriprasart T, Aragaki A, Baughman R, et al. A single US center experience of transbronchial lung cryobiopsy for diagnosing interstitial lung disease with a 2-scope technique. J Bronchology Interv Pulmonol 2017; 24: 131-135. 